\title{
Inhibitory Effects of Myricetrin and Dihydromyricetin toward $\alpha$-Glucosidase and Pancreatic Lipase with Molecular Docking Analyses and Their Interaction
}

\author{
Siyuan Mi, ${ }^{1,2}$ Jia Liu, ${ }^{3}$ Xiaojing Liu $\mathbb{D}^{1},{ }^{1}$ Yishan Fu, ${ }^{1}$ Junjie Yi $\mathbb{D}^{1},{ }^{1}$ and Shengbao Cai $\mathbb{D}^{1}$ \\ ${ }^{1}$ Faculty of Agriculture and Food, Yunnan Institute of Food Safety, Kunming University of Science and Technology, \\ Kunming 650500, Yunnan Province, China \\ ${ }^{2}$ Zhongshan School of Medicine, Sun Yat-sen University, Guangzhou 510000, Guangdong Province, China \\ ${ }^{3}$ Beijing Key Laboratory of the Innovative Development of Functional Staple and the Nutritional Intervention \\ for Chronic Disease, China National Research Institute of Food and Fermentation Industries Co. Ltd., Beijing 100015, China \\ Correspondence should be addressed to Junjie Yi; junjieyi@kust.edu.cn and Shengbao Cai; caikmust2013@kmust.edu.cn
}

Received 22 March 2021; Revised 24 May 2021; Accepted 17 July 2021; Published 27 July 2021

Academic Editor: Antoni Szumny

Copyright $\odot 2021$ Siyuan Mi et al. This is an open access article distributed under the Creative Commons Attribution License, which permits unrestricted use, distribution, and reproduction in any medium, provided the original work is properly cited.

\begin{abstract}
The aim of the current study was to evaluate the interaction effects of myricetrin and dihydromyricetin in inhibiting $\alpha$-glucosidase and pancreatic lipase at different combination ratios and concentrations and to illuminate the underlying mechanisms of their inhibitions by molecular docking analyses. Results showed that both phenolic compounds possessed good inhibitory effects toward two enzymes in a dose-dependent manner. Myricetrin demonstrated a stronger inhibition against $\alpha$-glucosidase (IC ${ }_{50}$, $41.14 \pm 2.52$ and more than $200 \mu \mathrm{g} / \mathrm{mL}$, respectively), while dihydromyricetin had a better pancreatic lipase inhibition (IC 50 , $244.96 \pm 4.24$ and $373.26 \pm 21.36 \mu \mathrm{g} / \mathrm{mL}$, respectively). Different interaction types were observed when myricetrin and dihydromyricetin inhibited $\alpha$-glucosidase and pancreatic lipase in combination, which were closely related to the combination ratio and concentration. For $\alpha$-glucosidase inhibition, synergistic effects were observed at relative low concentrations when the combination ratio of myricetrin to dihydromyricetin was set as $1: 2$, while strong synergistic effects existed at relative high concentrations for pancreatic lipase inhibition. In other combination ratios (1:1 or $2: 1)$, additive and/or antagonistic effects occurred. Molecular docking analyses showed that myricetrin formed nine hydrogen bonds with $\alpha$-glucosidase, while only three hydrogen bonds were formed between dihydromyricetin and $\alpha$-glucosidase. However, these two phenolic compounds had similar hydrogen bonds and hydrophobic interactions with pancreatic lipase. The present study suggested that myricetrin and dihydromyricetin or food materials rich in these two phenolic compounds could be exploited as $\alpha$-glucosidase and/or pancreatic lipase inhibitors to deal with health problems caused by excessive energy intake, and the combination ratio and concentration of these two phenolic compounds should be considered when producing new functional foods.
\end{abstract}

\section{Introduction}

Along with the improvement of living standards and the enrichment of material conditions, the diet pattern with high sugar and high fat has become more common, coupled with the sedentary lifestyle with little exercise, resulting in a serious excess of energy intake and thereby leading to the prevalence of many chronic diseases, such as obesity, diabetes, fatty liver, and cardiovascular disease $[1,2]$. These chronic diseases have caused heavy burden to the medical system all over the world. Therefore, how to effectively deal with these chronic diseases has become an urgent problem to be solved, which is also a hot and difficult research topic in many disciplines. Dietary intervention to reduce the risk of these chronic diseases is considered to be an effective approach [2-4]. Therefore, many food and nutrition scientists have put a lot of substantial efforts to prevent these chronic diseases.

Carbohydrates and fats in food are the main contributors to the body's energy intake, and therefore reducing 
carbohydrate and/or fat intake is considered an effective way to suppress energy intake [5-7]. In the digestive system, carbohydrates and fats need to be digested before they can be absorbed. As for carbohydrates, $\alpha$-glucosidase is one of the main enzymes for their digestion, and therefore, inhibiting $\alpha$-glucosidase can not only reduce energy intake derived from carbohydrate but also effectively control postprandial blood glucose of diabetic patients $[8,9]$. And for fats in food, pancreatic lipase is the main digestive enzyme, and thus, inhibiting pancreatic lipase is considered an effective method to reduce fat absorption [10,11]. Clinically, acarbose and orlistat are inhibitors of $\alpha$-glucosidase and pancreatic lipase, respectively. Although these clinical drugs are effective, they all have serious side effects. A large number of previous studies have found that many secondary metabolites of edible plants, especially phenolic compounds, have good inhibitory effects toward $\alpha$-glucosidase and/or pancreatic lipase $[6,7,10,12,13]$.

Phenolic compounds in plants are generally polyphenols, which contain multiple hydroxyl groups on the benzene ring. A certain edible plant commonly contains a variety of phenolic substances, and these phenolics often interact with each other when they exert their biological activities, such as synergistic or antagonistic effects $[6,10]$. Our previous study about the different phenolic extracts from palm fruits inhibiting pancreatic lipase and $\alpha$-glucosidase found that the main phenolics catechin and caffeic acid display complex interactions ranging from antagonistic effect to synergistic effect [6]. Moreover, a previous study also found that myricetrin and dihydromyricetin were the most predominant components of vine tea [14], and it is reported that vine tea has a good inhibitory effect toward pancreatic lipase or $\alpha$-glucosidase $[15,16]$. However, it is not clear how these two predominant phytochemical compounds interact with each other in inhibiting pancreatic lipase and/or $\alpha$-glucosidase. In addition, the investigation of the underlying mechanism about their enzyme inhibition is also scarce. Therefore, in the present work, the interaction effects of myricetrin and dihydromyricetin in inhibiting pancreatic lipase and $\alpha$-glucosidase were evaluated and the underlying mechanisms were illuminated by molecular docking analyses.

\section{Materials and Methods}

2.1. Materials and Chemical Reagent. Porcine pancreatic lipase (EC: $3.1 .1 .3,127 \mathrm{U} / \mathrm{mg}$ ), $\alpha$-glucosidase of Saccharomyces cerevisiae (EC: $3.2 .1 .20 ; \geq 10$ units/mg protein Type I, lyophilized powder), $p$-nitrophenyl laurate, and $p$-nitrophenyl- $\alpha$-D-glucopyranoside ( $p$ NPG, purity $\geq 99.0 \%$ ) were obtained from Sigma (Sigma-Aldrich, Shanghai, China). The standards of myricetrin and dihydromyricetin (purity $\geq 98.0 \%$ ) were purchased from Chengdu Must Bio-Technology Co., Ltd. (Chengdu, Sichuan, China). Other reagents used were of analytical grade.
2.2. $\alpha$-Glucosidase Inhibition Assay. The inhibitory effects of myricetrin and dihydromyricetin toward $\alpha$-glucosidase were measured according to the published method $[13,17]$ with minor modifications. The concentrations of $\alpha$-glucosidase solution and $p$ NPG (the substrate) in the present study were $2.0 \mathrm{U} / \mathrm{mL}$ and $5.0 \mathrm{mM}$, respectively. Myricetrin and dihydromyricetin were dissolved in dimethyl sulfoxide (DMSO) as stock solution and then diluted with the reaction buffer solution (PBS, $\mathrm{pH}$ 6.8) to the appropriate concentration (finally DMSO $<0.5 \%$ ). A SpectraMax M5 microplate reader (Molecular Device, Sunnyvale, CA, USA) was used to measure the absorbance of each sample at $405 \mathrm{~nm}$, and the inhibitory ratio of each sample was calculated by the following formula: Inhibitory ratio $(\%)=(1-$ OD of sample/ OD of control) $\times 100 \%$.

2.3. Pancreatic Lipase Inhibition Assay. The inhibitory effects of these two phenolic standards toward pancreatic lipase were measured according to the method reported earlier $[13,17]$ with some modifications. The concentration of porcine pancreatic lipase solution in the current study was $5 \mathrm{mg} / \mathrm{mL}$. The sample was dissolved in DMSO and then diluted with the reaction buffer (Tris- $\mathrm{HCl}$ buffer, $\mathrm{pH} 8.0$, finally DMSO $<0.5 \%$ ). The absorbance (OD value) at $400 \mathrm{~nm}$ was measured using a SpectraMax M5 microplate reader. The inhibition ratio of pancreatic lipase activity was determined as follows: Inhibitory ratio $(\%)=(1-$ OD of sample/OD of control) $\times 100 \%$.

2.4. Interaction of Myricetrin and Dihydromyricetin in $\alpha$-Glucosidase and Pancreatic Lipase Inhibition. CalcuSyn software (Biosoft, Ferguson, MO, USA) was applied to analyze the interaction type of myricetrin and dihydromyricetin in $\alpha$-glucosidase and pancreatic lipase inhibition. The dose ratios between myricetrin and dihydromyricetin were set as $1: 1,1: 2$, and $2: 1$, respectively. The tested method of inhibitory activity was completely consistent with the above corresponding method.

2.5. Molecular Docking. In order to illuminate the underlying mechanisms of these two phenolic compounds inhibiting lipase and $\alpha$-glucosidase, molecular docking analyses of myricetrin and dihydromyricetin with lipase and glycosidase, respectively, were performed by using SYBYL-X 2.1.1 software (Tripos, Inc., St. Louis, MO, USA), and Surflex-Dock Geom was applied as the docking mode. The 3D structure of myricetrin and dihydromyricetin were downloaded from the NCBI database (http://www.ncbi.nlm. nih.gov/pccompound), and the 3D structure of lipase (PDB Code: 1ETH) were obtained from the RCSB protein database (http://www.rcsb.org/pdb/home.do). Since the crystal structure of $\alpha$-glucosidase derived from $S$. cerevisiae was not available yet and the sequence of isomaltase derived from $S$. cerevisiae is very similar to that of $\alpha$-glucosidase from the 
same source, isomaltase (PDB Code: 3A4A) was used for molecular docking analysis in the present work, which was consistent with the previous publication [18]. The highprecision mode (SFXC) was used for docking assay. Before docking, 1ETH and 3A4A were optimized to remove water molecules, metal ions, and their own ligands, and then the protein was hydrogenated and charged. $C$-scores and $T$ scores were used as indicators for identifying the optimal analytical result.

2.6. Statistical Analysis. Data in the current work are presented as mean values $(n=3) \pm$ standard deviation (SD) and analyzed by one-way ANOVA. Tukey's procedure was applied to determine the significance of the differences $(p<0.05)$. All analyses were performed by using Origin 8.5 software (OriginLab, Northampton, MA, USA).

\section{Results and Discussion}

3.1. $\alpha$-Glucosidase Inhibition of Myricetrin and Dihydromyricetin. In the gastrointestinal tract, $\alpha$-glucosidase is one of the most vital carbohydrate digestive enzymes. Inhibiting $\alpha$-glucosidase activity has been regarded as an effective way to control postprandial blood glucose in diabetic patients [9], which is also the action mechanism of the clinical drug acarbose. In this study, the $\alpha$-glucosidase inhibition results of myricetrin and dihydromyricetin are presented in Figure 1. Both myricetrin and dihydromyricetin displayed good inhibitory effects toward $\alpha$-glucosidase in a dose-dependent manner at concentrations ranging from $25 \mu \mathrm{g} / \mathrm{mL}$ to $125 \mu \mathrm{g} / \mathrm{mL}$. Compared with dihydromyricetin, myricetrin possessed a much stronger inhibition toward $\alpha$-glucosidase at all tested concentrations $(p<0.05)$. At the lowest tested concentration $(25 \mu \mathrm{g} / \mathrm{mL})$, the inhibitory ratio of myricetrin was about 9 times as much as that of dihydromyricetin. When tested at $125 \mu \mathrm{g} / \mathrm{mL}$, the inhibitory ratio of myricetrin was approximately $90 \%$, while the inhibitory ratio of dihydromyricetin was no more than $30 \%$. The $\mathrm{IC}_{50}$ values of myricetrin and dihydromyricetin were $41.14 \pm 2.52 \mu \mathrm{g} / \mathrm{mL}$ and more than $200 \mu \mathrm{g} / \mathrm{mL}$, respectively. The difference between these two phenolic compounds in the inhibitory capacity toward $\alpha$-glucosidase may be mainly caused by their structural differences. A previous study also reported that flavonoids with different structures displayed great differences in the $\alpha$-glucosidase inhibition [19]. Moreover, dietary phenolic compounds could cooperate with acarbose to inhibit $\alpha$-glucosidase activity [20]. In the current study, the $\mathrm{IC}_{50}$ value of acarbose was $0.56 \pm 0.08 \mu \mathrm{g} /$ $\mathrm{mL}$.

3.2. Pancreatic Lipase Inhibition of Myricetrin and Dihydromyricetin. In the intestinal tract, pancreatic lipase is one of the key enzymes responsible for fat digestion, and inhibition of this enzyme can effectively reduce fat absorption, thereby preventing obesity and other related health problems $[10,13]$. As shown in Figure 2, both myricetrin and dihydromyricetin displayed good inhibitory effects toward pancreatic lipase in a dose-dependent response. Unlike the

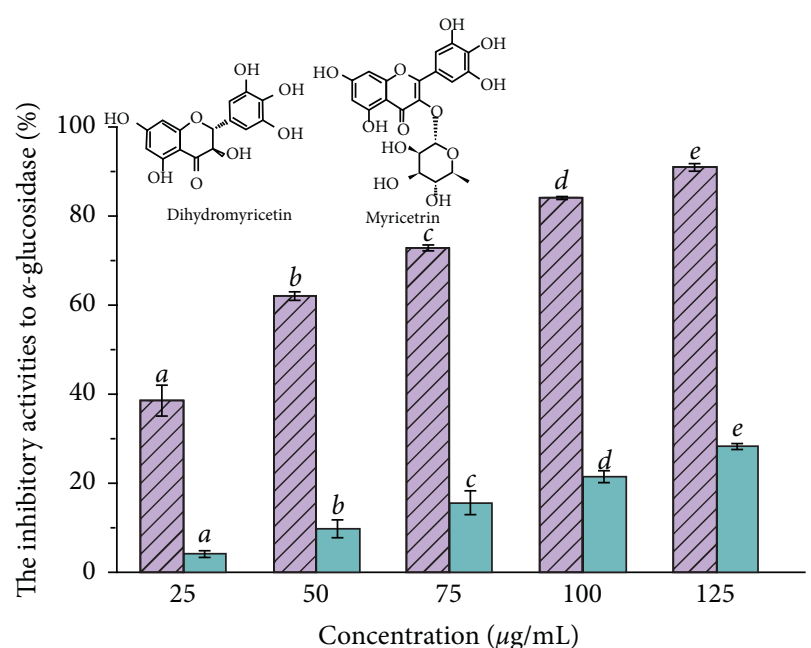

QZA Myricetrin

Dihydromyricetin

FIgURE 1: Inhibition effect of myricetrin and dihydromyricetin toward $\alpha$-glucosidase. All values are expressed as mean $\pm \mathrm{SD}$ $(n=3)$. Different letters indicate significant differences at $p<0.05$.

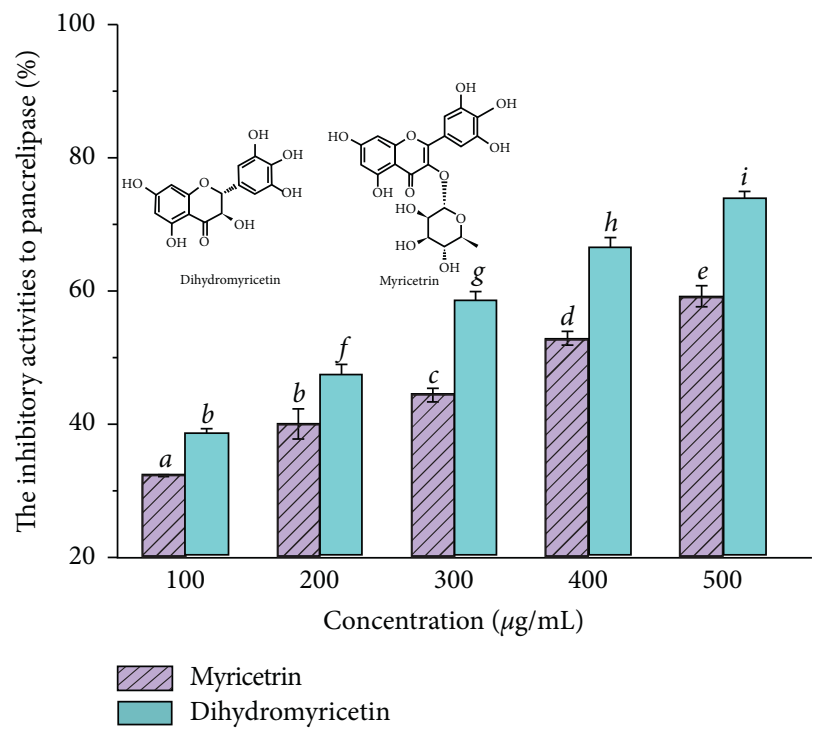

FIGURE 2: Inhibition effect of myricetrin and dihydromyricetin against pancreatic lipase. All values are expressed as mean \pm SD $(n=3)$. Different letters indicate significant differences at $p<0.05$.

phenomenon observed in the $\alpha$-glucosidase inhibition, dihydromyricetin showed better inhibitory effects toward pancreatic lipase than myricetrin at all tested concentrations ranging from $100 \mu \mathrm{g} / \mathrm{mL}$ to $500 \mu \mathrm{g} / \mathrm{mL}(p<0.05)$. In the present work, the $\mathrm{IC}_{50}$ values of myricetrin and dihydromyricetin were $373.26 \pm 21.36 \mu \mathrm{g} / \mathrm{mL}$ and $244.96 \pm 4.24 \mu \mathrm{g} / \mathrm{mL}$, respectively. When the concentration was $100 \mu \mathrm{g} / \mathrm{mL}$, the inhibition ratios were approximately $31 \%$ and $38 \%$ for myricetrin and dihydromyricetin, respectively, and the inhibition ratios increased to $84 \%$ and $92 \%$, respectively, when the concentration was $500 \mu \mathrm{g} / \mathrm{mL}$. As aforementioned in $\alpha$-glucosidase inhibition, the inhibitory capacities of phenolic compounds toward a certain 
enzyme may be closely dependent on their chemical structures. A previous study about the lipase inhibition of different tea polyphenols also found that the inhibitory capacities varied with the polyphenolic structures, and the structure-activity relationship was further analyzed by 3D-QSAR models [21].

\subsection{Interaction of Myricetrin and Dihydromyricetin on In-} hibition of $\alpha$-Glucosidase and Pancreatic Lipase. Some previous reports have confirmed that phenolic compounds may interact with each other when inhibiting lipase or $\alpha$-glycosidase, such as antagonistic effect, additive effect, or synergistic effect $[6,10]$. Moreover, the type of interaction commonly depended on the concentration and proportion of phenolic substances [6]. In this study, the interactions of myricetrin and dihydromyricetin in inhibiting pancreatic lipase and $\alpha$-glucosidase were determined by CalcuSyn software with three different combination ratios $(1: 1,1: 2$, and 2:1), and results are summarized in Tables 1 and 2, respectively.

Table 1 presents the interaction types of myricetrin and dihydromyricetin on $\alpha$-glucosidase inhibition at three different ratios. When the ratio was set at $1: 1$, the inhibitory capacities increased from $12.80 \pm 1.53 \%$ to $76.68 \pm 0.62 \%$ and CI values gradually decreased from 1.313 to 0.958 with the concentration of myricetrin increasing from 12.5 to $62.5 \mu \mathrm{g} /$ $\mathrm{mL}$, suggesting that the interaction type between myricetrin and dihydromyricetin changed from antagonistic (low concentration) to additive effect (high concentration). When the ratio of myricetrin to dihydromyricetin was set as $1: 2$, the inhibitory ratio was $16.80 \pm 2.19 \%$ at the myricetrin concentration of $8.3 \mu \mathrm{g} / \mathrm{mL}$ and then increased by more than 3 times when the concentration of myricetrin was $41.6 \mu \mathrm{g} /$ $\mathrm{mL}$. The CI values increased from 0.821 to 0.929 , indicating that the interaction type changed from synergistic effect to additive effect. However, when myricetrin and dihydromyricetin were combined at a ratio of $2: 1$, the CI values increased from 0.577 to 1.130 , indicating that the interaction effect of these two phenolic compounds was synergistic effect at the low concentration but changed to antagonistic effect at the high concentration. It is worth noting that the combination of myricetrin and dihydromyricetin at a ratio of $2: 1$ showed stronger inhibitory effects toward $\alpha$-glucosidase when compared with the combination at ratio of $1: 1$ or $1: 2$, especially at a relative low concentration. This phenomenon may be due to the much stronger $\alpha$-glucosidase inhibitory effect of myricetrin. Similar phenomena were observed by some previous studies $[6,10]$. For example, a previous study also found that different flavonoids isolated from Moringa oleifera leaf showed synergistic inhibition on $\alpha$-glucosidase activity [22].

The interaction results of myricetrin and dihydromyricetin on pancreatic lipase are presented in Table 2 . When myricetrin and dihydromyricetin were combined at the ratio of $1: 1$, the CI value decreased with the increasing concentration. An antagonistic effect was observed at the concentrations of 50 and $100 \mu \mathrm{g} / \mathrm{mL}$, and an additive effect was observed at the concentrations of 150 and $200 \mu \mathrm{g} / \mathrm{mL}$, while a synergistic effect was observed at the concentration of $250 \mu \mathrm{g} / \mathrm{mL}$. When the ratio of myricetrin to dihydromyricetin was set as $1: 2$, the interaction type was additive at the myricetrin concentration of $33.3 \mu \mathrm{g} / \mathrm{mL}$, while the interaction type was always synergistic at other tested concentrations according to the CI values. However, when the combined ratio was set as $2: 1$, CI values were always more than 1.10 at all tested concentrations, indicating that the antagonistic effect always existed at this combination ratio. Among these three combination doses, the ratio of myricetrin to dihydromyricetin at $1: 2$ possessed the strongest inhibitory effect toward pancreatic lipase, which was consistent with the stronger inhibitory capacity of dihydromyricetin. The current result was similar to the previous finding [10]. Cai et al. also found that the binary combination of caffeic acid, ferulic acid, and $p$-coumaric acid also exhibited synergistic effects in inhibiting pancreatic lipase only at relative higher concentrations [10]. Moreover, a previous study found that a phenolic compound kaempferol could exhibit synergistic effects with orlistat to inhibit lipase [11].

\subsection{Molecular Docking Results of $\alpha$-Glucosidase and Pan-} creatic Lipase. The enzyme inhibitory mechanisms of myricetrin and dihydromyricetin on $\alpha$-glucosidase and pancreatic lipase were illuminated by the molecular docking method with the computational modeling software SYBYL$\mathrm{X}$ 2.1.1, and the hydrophobic interactions were analyzed by Ligplot+ software. The related parameters of the docking scores, hydrogen bond parameters, and hydrophobic effects of these standards with $\alpha$-glucosidase or pancreatic lipase are summarized in Tables 3 and 4, respectively. The parameters in Tables 3 and 4 displayed the detailed information of the receptor-ligand interactions, including reliability, charge, van der Waals force, hydrogen bond, lipophilic, and so on. Among these parameters, $C$-scores and $T$-scores are the most vital parameters, which reflect the reliability of docking results and binding affinity ability between receptor and ligand, respectively.

The docking results of myricetrin and dihydromyricetin with $\alpha$-glucosidase are summarized in Table 3 and Figure 3. $C$-score values of myricetrin and dihydromyricetin were four, indicating that the docking results of these two phenolic compounds with $\alpha$-glucosidase were credible. $T$-score values of myricetrin and dihydromyricetin were 4.1619 and 4.6978, respectively, suggesting that dihydromyricetin may bind more tightly to $\alpha$-glucosidase than myricetrin. For myricetrin, it formed nine hydrogen bonds with seven amino acid residues, and the longest and shortest hydrogen bonds were $2.542 \AA$ (formed with Asp352) and $1.666 \AA$ (formed with Tyr158), respectively. However, dihydromyricetin just formed three hydrogen bonds with three amino acid residues, and the longest and shortest hydrogen bonds were $2.144 \AA$ (formed with Gln353) and $1.908 \AA$ (formed with Asp215), respectively. A previous study has pointed out that the number and distance of hydrogen bonds played important roles for inhibitors to inhibit enzyme activity [7]. In the present work, although the average distance of hydrogen bonds of these two phenolic 
TABLE 1: Interaction between myricetrin and dihydromyricetin on $\alpha$-glucosidase inhibition.

\begin{tabular}{|c|c|c|c|c|c|c|c|c|}
\hline Ratio & \multicolumn{2}{|c|}{$\begin{array}{l}\text { Myricetrin Dihydromyricetin } \\
\text { Tested concentration }(\mu \mathrm{g} / \mathrm{mL})\end{array}$} & \multirow{2}{*}{$\begin{array}{c}\alpha \text {-Glucosidase inhibition (\%) } \\
12.80 \pm 1.53\end{array}$} & \multirow{2}{*}{$\begin{array}{c}\mathrm{CI}^{\mathrm{a}} \\
1.313\end{array}$} & \multirow{2}{*}{$\frac{\mathrm{Fa}^{\mathrm{b}}(\%)}{25.0}$} & \multirow{2}{*}{$\begin{array}{c}\text { CI for } \mathrm{Fa} \\
1.236\end{array}$} & \multicolumn{2}{|c|}{$\begin{array}{c}\text { Myricetrin Dihydromyricetin } \\
\text { Calculated concentration } \\
(\mu \mathrm{g} / \mathrm{mL})\end{array}$} \\
\hline \multirow{5}{*}{$1: 1$} & 12.5 & 12.5 & & & & & 19.58 & 19.58 \\
\hline & 25.0 & 25.0 & $33.40 \pm 1.51$ & 1.225 & 50.0 & 1.101 & 34.45 & 34.45 \\
\hline & 37.5 & 37.5 & $52.48 \pm 1.94$ & 1.127 & 75.0 & 0.983 & 60.61 & 60.61 \\
\hline & 50.0 & 50.0 & $68.42 \pm 1.44$ & 0.992 & 90.0 & 0.880 & 106.64 & 106.64 \\
\hline & 62.5 & 62.5 & $76.68 \pm 0.62$ & 0.958 & - & - & - & - \\
\hline \multirow{5}{*}{$1: 2$} & 8.3 & 16.7 & $16.80 \pm 2.19$ & 0.821 & 25.0 & 0.825 & 11.47 & 22.94 \\
\hline & 16.7 & 33.3 & $37.34 \pm 2.27$ & 0.831 & 50.0 & 0.875 & 24.38 & 48.76 \\
\hline & 25.0 & 50.0 & $52.10 \pm 2.54$ & 0.851 & 75.0 & 0.932 & 51.84 & 103.68 \\
\hline & 33.3 & 66.7 & $60.54 \pm 2.01$ & 0.913 & 90.0 & 0.996 & 110.22 & 220.44 \\
\hline & 41.6 & 83.4 & $68.07 \pm 2.67$ & 0.929 & - & - & - & - \\
\hline \multirow{5}{*}{$2: 1$} & 16.7 & 8.3 & $44.26 \pm 3.36$ & 0.577 & 25.0 & 0.438 & 7.46 & 3.73 \\
\hline & 33.3 & 16.7 & $54.62 \pm 1.10$ & 0.892 & 50.0 & 0.727 & 24.22 & 12.11 \\
\hline & 50.0 & 25.0 & $62.19 \pm 1.03$ & 1.107 & 75.0 & 1.207 & 78.66 & 39.33 \\
\hline & 66.7 & 33.3 & $72.47 \pm 1.17$ & 1.109 & 90.0 & 2.007 & 255.28 & 127.64 \\
\hline & 83.3 & 41.7 & $78.61 \pm 3.92$ & 1.130 & - & - & - & - \\
\hline
\end{tabular}

${ }^{\mathrm{a}} \mathrm{CI}$, combination index. $\mathrm{CI} \leq 0.90,0.90<\mathrm{CI}<1.10$, and $\mathrm{CI} \geq 1.10$ indicate synergistic, additive, and antagonistic effects, respectively; ${ }^{\mathrm{b}} \mathrm{Fa}$, fraction affected by the combination dose of myricetrin and dihydromyricetin.

TABLE 2: Interaction between myricetrin and dihydromyricetin on pancreatic lipase inhibition.

\begin{tabular}{|c|c|c|c|c|c|c|c|c|}
\hline Ratio & \multicolumn{2}{|c|}{$\begin{array}{l}\text { Myricetrin Dihydromyricetin } \\
\text { Tested concentration }(\mu \mathrm{g} / \mathrm{mL})\end{array}$} & \multirow{2}{*}{$\begin{array}{c}\text { Pancreatic lipase inhibition (\%) } \\
28.33 \pm 1.69\end{array}$} & \multirow{2}{*}{$\begin{array}{c}\mathrm{CI}^{\mathrm{a}} \\
1.294\end{array}$} & \multirow{2}{*}{$\frac{\mathrm{Fa}^{\mathrm{b}}(\%)}{25.0}$} & \multirow{2}{*}{$\begin{array}{c}\text { CI for } \mathrm{Fa} \\
1.426\end{array}$} & \multicolumn{2}{|c|}{$\begin{array}{c}\text { Myricetrin Dihydromyricetin } \\
\text { Calculated concentration } \\
(\mu \mathrm{g} / \mathrm{mL})\end{array}$} \\
\hline \multirow{5}{*}{$1: 1$} & 50.0 & 50.0 & & & & & 44.39 & 44.39 \\
\hline & 100.0 & 100.0 & $42.39 \pm 2.24$ & 1.188 & 50.0 & 1.044 & 128.35 & 128.35 \\
\hline & 150.0 & 150.0 & $52.76 \pm 0.52$ & 1.064 & 75.0 & 0.801 & 371.17 & 371.17 \\
\hline & 200.0 & 200.0 & $60.61 \pm 2.82$ & 0.962 & 85.0 & 0.700 & 686.34 & 686.34 \\
\hline & 250.0 & 250.0 & $68.32 \pm 0.65$ & 0.800 & - & - & - & - \\
\hline \multirow{5}{*}{$1: 2$} & 33.3 & 66.6 & $31.25 \pm 2.41$ & 1.034 & 25.0 & 1.247 & 26.52 & 53.03 \\
\hline & 66.7 & 133.4 & $47.02 \pm 0.53$ & 0.858 & 50.0 & 0.778 & 70.68 & 141.37 \\
\hline & 100.0 & 200.0 & $58.40 \pm 0.63$ & 0.711 & 75.0 & 0.511 & 188.41 & 376.83 \\
\hline & 133.3 & 266.6 & $65.73 \pm 1.65$ & 0.636 & 85.0 & 0.410 & 332.36 & 664.71 \\
\hline & 166.7 & 333.4 & $74.46 \pm 0.41$ & 0.469 & - & - & - & - \\
\hline \multirow{5}{*}{$2: 1$} & 66.7 & 33.3 & $29.50 \pm 1.04$ & 1.552 & 25.0 & 1.547 & 62.67 & 31.33 \\
\hline & 133.3 & 66.7 & $38.70 \pm 1.34$ & 1.447 & 50.0 & 1.388 & 207.40 & 103.70 \\
\hline & 200.0 & 100.0 & $46.78 \pm 2.09$ & 1.423 & 75.0 & 1.291 & 686.42 & 343.21 \\
\hline & 266.7 & 133.3 & $55.93 \pm 1.14$ & 1.352 & 85.0 & 1.253 & 1372.44 & 686.22 \\
\hline & 333.3 & 66.7 & $60.92 \pm 1.23$ & 1.331 & - & - & - & - \\
\hline
\end{tabular}

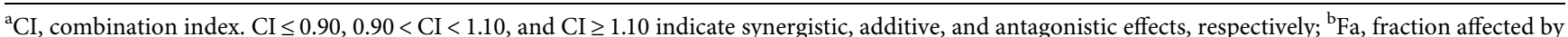
the combination dose of myricetrin and dihydromyricetin.

compounds were similar, myricetrin formed far more hydrogen bonds than dihydromyricetin, which may explain why myricetrin showed much stronger $\alpha$-glucosidase inhibition than dihydromyricetin (Figure 1). Both phenolic compounds formed a hydrogen bond with Gln353, suggesting that Gln 353 may be one of the amino acid residues that affect $\alpha$-glucosidase to exert its catalytic reaction. A previous study have reported that phloretin may inhibit $\alpha$-glucosidase by forming hydrogen bonds with five amino acid residues of $\alpha$-glucosidase, namely Asp69, Asp215, Arg442, Gln353, and Asn 350 [23]. Moreover, it is found that gallocatechin gallate can form a hydrogen bond with Arg315 to exert their inhibitory effect on $\alpha$-glucosidase [24]. In the present work, myricetrin was also found to form hydrogen bonds with Asp69, Arg315, and Gln353, and dihydromyricetin was observed to form hydrogen bonds with Asp215, Asn350, and Gln353. Besides hydrogen bonds, these two phenolic compounds were also found to form hydrophobic interactions with several amino acid residuals of $\alpha$-glucosidase (Table 3, Figures 3(d) and 3(h)). Many previous studies have pointed out that the hydrophobic interaction may play a critical role in the enzymatic inhibition of some inhibitors $[25,26]$.

As for the molecular docking analysis of pancreatic lipase (Table 4 and Figure 4), the $C$-scores of the myricetrin and dihydromyricetin were 5 and 4 , respectively, indicating that the docking results were reliable. And the T-scores of myricetrin and dihydromyricetin were 3.7803 and 3.8534, 
TABLE 3: Docking parameters, hydrogen bonds, and hydrophobic interactions observed between flavonoids and $\alpha$-glucosidase from molecular docking simulation analysis.

\begin{tabular}{lcc}
\hline & Myricetrin & Dihydromyricetin \\
\hline -score & 4 & 4 \\
$T$-score & 4.1619 & 4.6978 \\
PMF-score & -192.4817 & -126.1538 \\
CHEM-score & -32.1584 & -23.1931 \\
G-score & -306.987 & -162.9552 \\
D-sore & -212.4343 & -165.0354 \\
Number of hydrogen interactions & 9 & 3 \\
Amino acid residues involved in & Arg315, Gln353, Asp352, Arg213, Asp69, & Asp215, Asn350, Gln353 \\
hydrogen bonds & Tyr158, Asn415 & Asn350, Gln353 \\
H-bond donor & Arg315, Gln353, Asn415, Arg213, Asp69 & Asp215 \\
H-bond receptor & Asp352, Tyr158 & 2.0623 \\
Average distance $(\AA)$ & 2.1720 & 10 \\
Number of hydrophobic interactions & 9 & Asp307, Arg315, Phe159, Tyr158, Glu411, \\
Amino acid residues involved in & Phe303, Gln353, Asp215, Tyr158, Phe178, Glu277, \\
hydrophobic interactions & Phe178, Tyr72, Arg442, Phe303 & His112, Tyr72, Val216, Asp352 \\
\hline
\end{tabular}

TABle 4: Docking parameters, hydrogen bonds, and hydrophobic interactions observed between flavonoids and pancreatic lipase from molecular docking simulation analysis.

\begin{tabular}{lcc}
\hline & Myricetrin & Dihydromyricetin \\
\hline C-score & 5 & 4 \\
$T$-score & 3.7803 & 3.9067 \\
PMF-score & -105.3467 & -79.0799 \\
CHEM-score & -26.5111 & -24.1416 \\
G-score & -231.122 & -171.9494 \\
D-sore & -164.596 & -131.2446 \\
Number of hydrogen interactions & 3 & 3 \\
Amino acid residues involved in & Gly77, Asp80, Phe216 & Gly77, Asp80, Tyr115 \\
hydrogen bonds & Gly77 & Gly77 \\
H-bond donor & Asp80, Phe216 & Asp80, Tyr115 \\
H-bond receptor & 2.2590 & 2.3867 \\
Average distance $(\AA)$ & 10 & 10 \\
Number of hydrophobic interactions & Phe78, Ile79, Asp80, Tyr115, Ser153, Ala179, \\
Amino acid residues involved in & Pro181, Val260, Ala261, His264 & \\
hydrophobic interactions & & Psp80, Tyr115, Ser153, Ala179, Pro181, \\
\hline
\end{tabular}

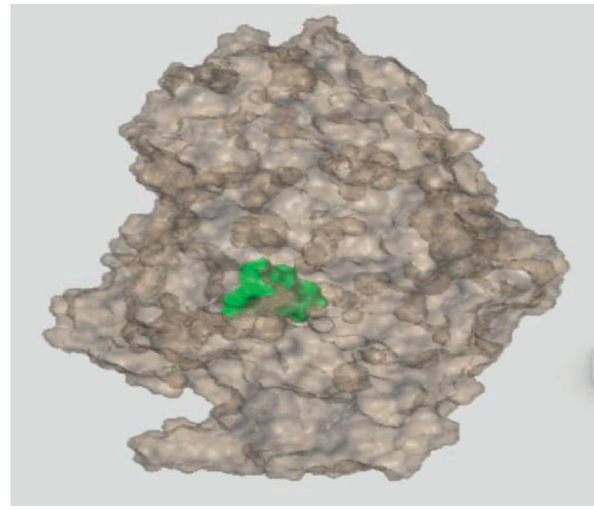

(a)

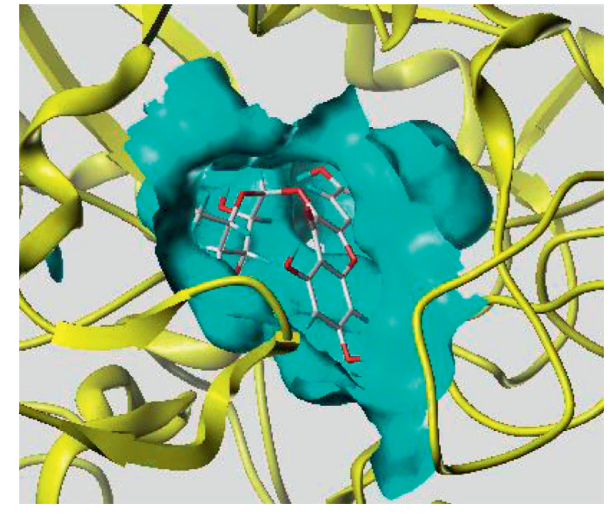

(b)

Figure 3: Continued. 


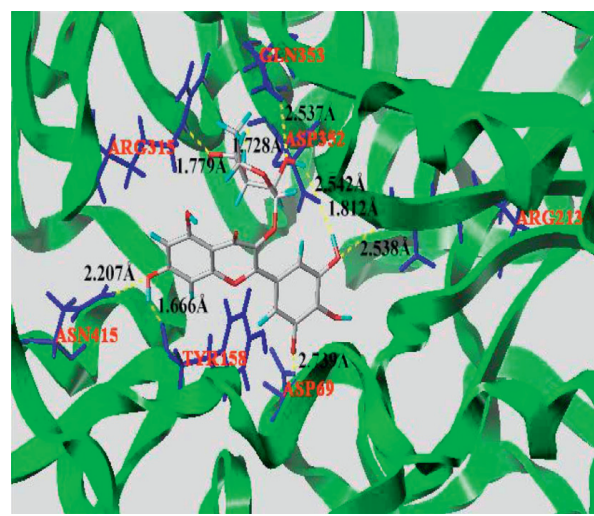

(c)

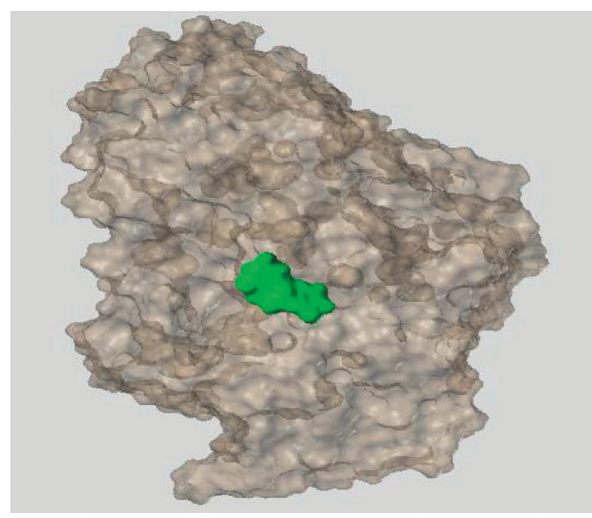

(e)

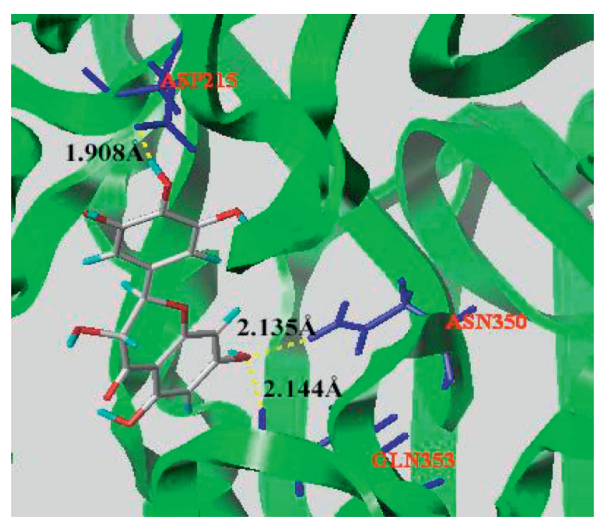

(g)

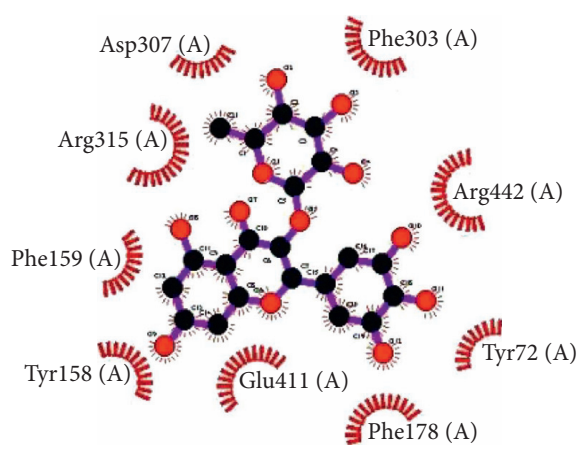

(d)

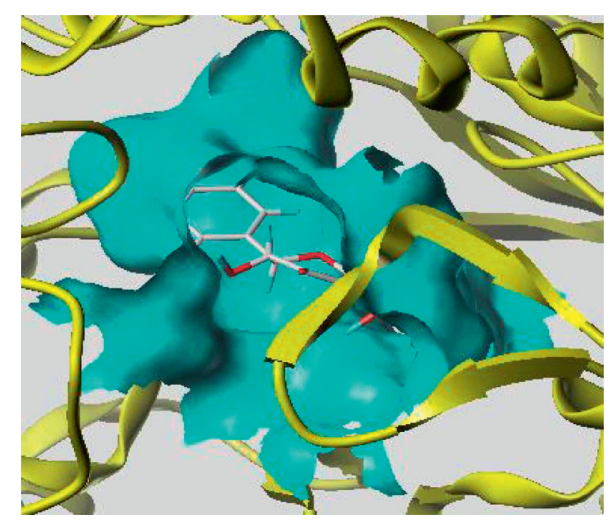

(f)

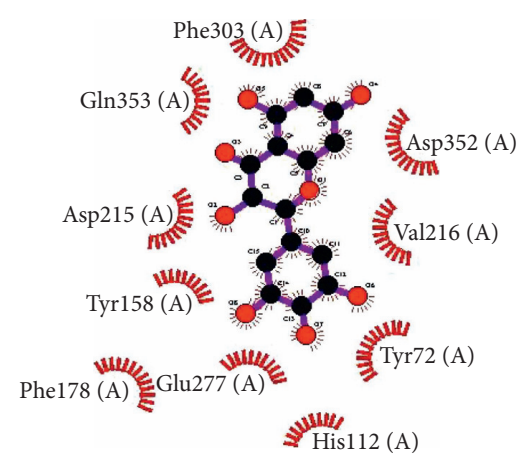

(h)

Figure 3: The molecule docking results of myricetrin (a-d) and dihydromyricetin (e-h) with $\alpha$-glucosidase.

respectively, suggesting that these two phenolic compounds have a similar affinity with pancreatic lipase. As shown in Figure 4(c), myricetrin could form three hydrogen bonds with three amino acid residues, namely Gly77, Phe216, and Asp80, and the longest and shortest distances of hydrogen bonds were $2.360 \AA$ (formed with Asp80) and $2.179 \AA$ (formed with Gly77), respectively, with an average distance of $2.259 \AA$. Dihydromyricetin also formed three hydrogen bonds with three amino acid residues, namely Gly77,
Tyr115, and Asp80, and the longest and shortest distances of hydrogen bonds were $2.396 \AA$ (formed with Asp80) and $2.380 \AA$ (formed with Gly77), respectively, with an average distance of $2.3867 \AA$. In addition, both of these phenolic compounds also formed ten hydrophobic interactions with ten amino acid residues of pancreatic lipase, and eight of the ten amino acid residues are the same. According to the docking result, it is reasonable to speculate that the difference in pancreatic lipase inhibitory activity between 


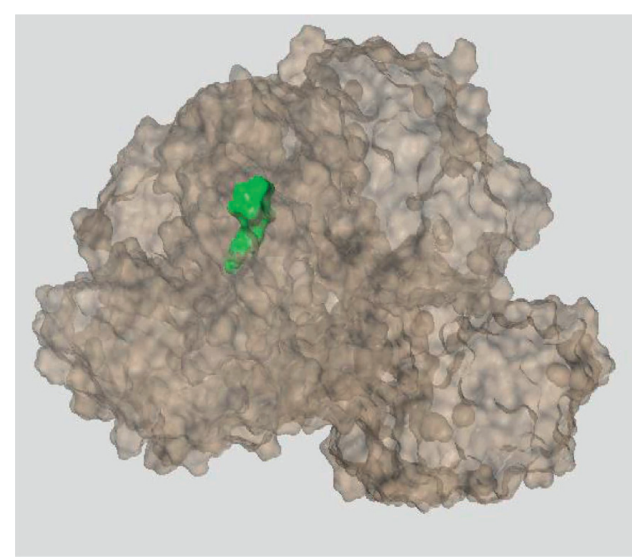

(a)

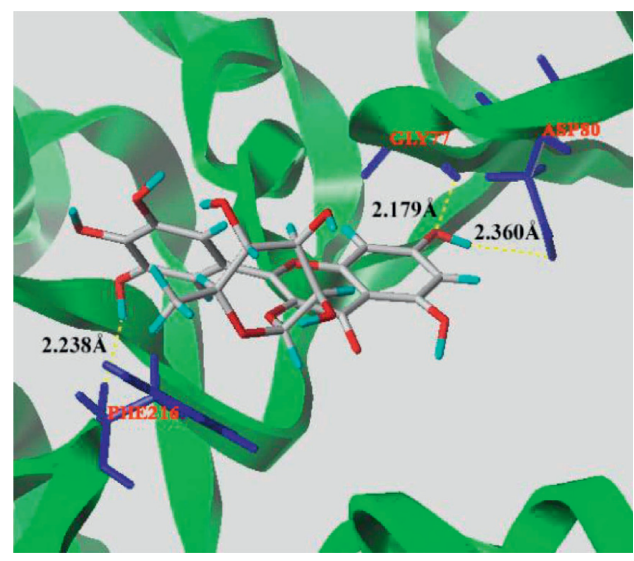

(c)

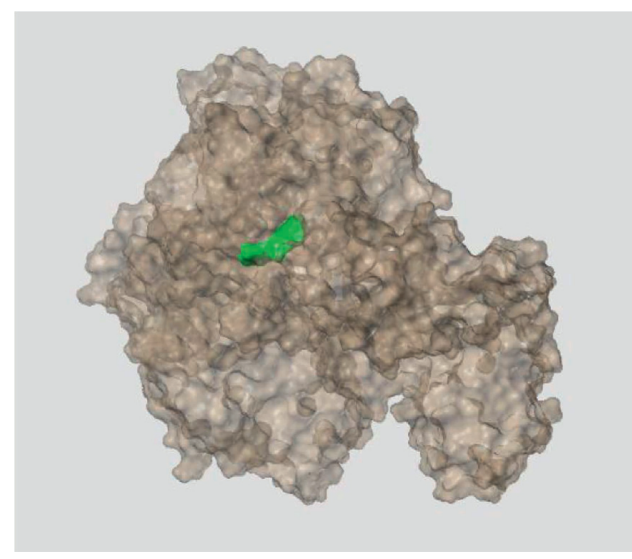

(e)

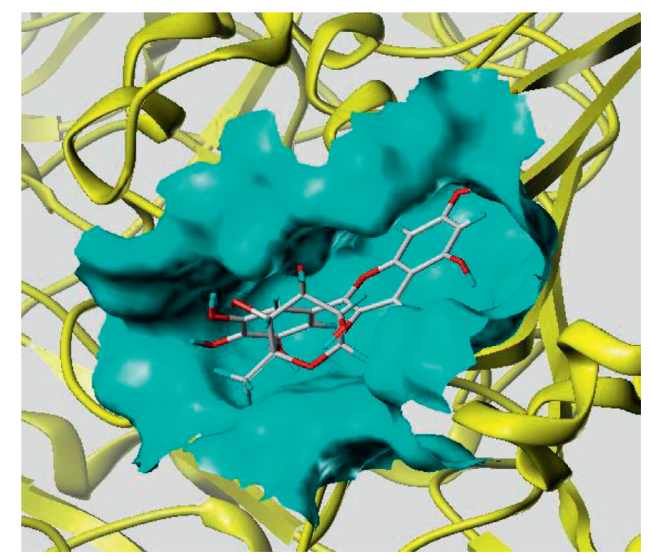

(b)

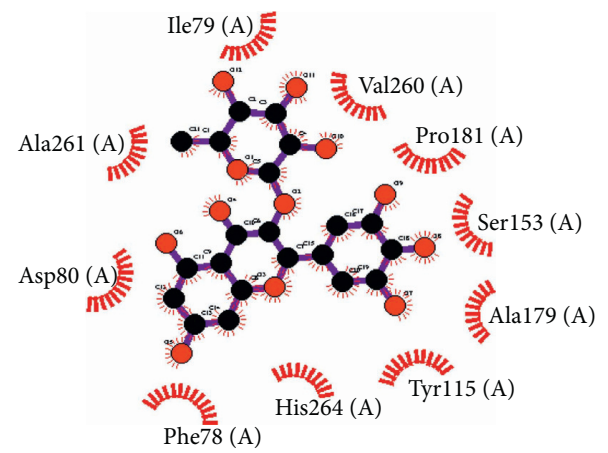

(d)

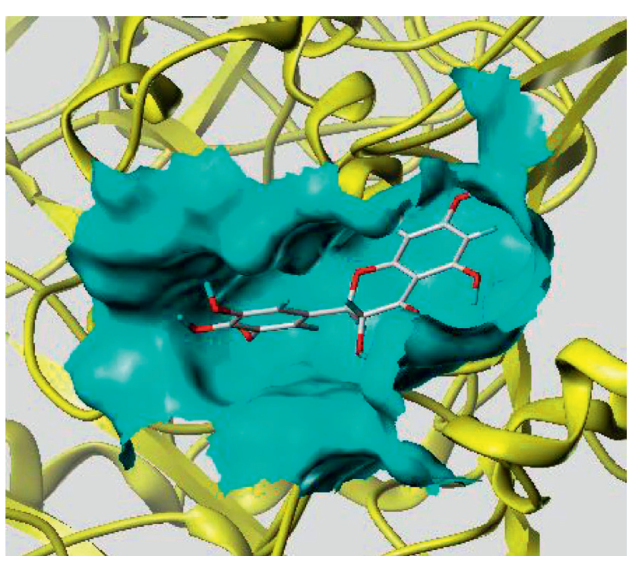

(f)

Figure 4: Continued. 


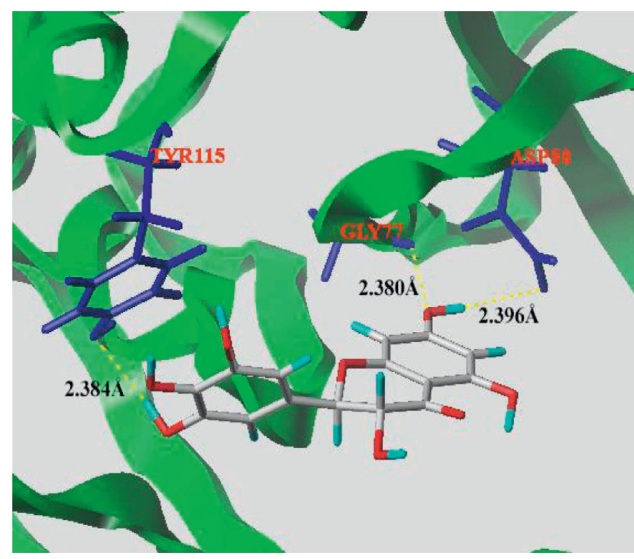

(g)

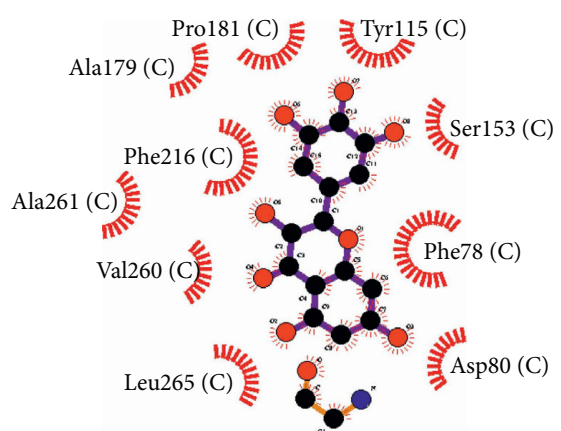

(h)

FIGURE 4: The molecular docking results of myricetrin $(\mathrm{a}-\mathrm{d})$ and dihydromyricetin $(\mathrm{e}-\mathrm{h})$ with pancreatic lipase.

myricetrin and dihydromyricetin may be due to the slightly different amino acid residues they interacted with in hydrogen bonds and hydrophobic interactions.

\section{Conclusions}

In the current study, results showed that both myricetrin and dihydromyricetin exhibited good inhibitory effects toward $\alpha$-glucosidase and pancreatic lipase in a dose-dependent manner. Myricetrin showed a much stronger $\alpha$-glucosidase inhibitory effect than dihydromyricetin. However, dihydromyricetin had a better inhibitory activity against pancreatic lipase than myricetrin. Different interaction types existed when myricetrin and dihydromyricetin inhibited $\alpha$-glucosidase and pancreatic lipase in combination, which were closely dependent on the combination ratio and concentration of these two phenolic compounds. For $\alpha$-glucosidase inhibition, synergistic effects were observed at relative low concentrations when the combination ratio of myricetrin to dihydromyricetin was set as $1: 2$. However, for pancreatic lipase inhibition, strong synergistic effects were found at relative high concentrations when the combination ratio of myricetrin to dihydromyricetin was set as $1: 2$. In other combination ratios $(1: 1$ or $2: 1)$, additive and/or antagonistic effects were occurred. Molecular docking results indicated that forming more hydrogen bonds between myricetrin and $\alpha$-glucosidase may be the reason that myricetrin had a stronger $\alpha$-glucosidase inhibitory activity than dihydromyricetin. These two phenolic compounds had similar hydrogen bonds and hydrophobic interactions with pancreatic lipase only with a slight difference of interacted amino acid residues. The present study indicated that myricetrin and dihydromyricetin or food materials rich in these two phenolic compounds could be developed as $\alpha$-glucosidase and/or pancreatic lipase inhibitors to deal with health issues caused by excessive energy intake, and the combination ratio and concentration of these two phenolic compounds should be taken into consideration when producing new functional foods.

\section{Data Availability}

All data included in this study are available upon request by contacting the corresponding author.

\section{Conflicts of Interest}

There are no conflicts of interest regarding the publication of this article.

\section{Authors' Contributions}

Siyuan Mi and Jia Liu contributed equally to this work.

\section{Acknowledgments}

This study was financially supported by the Applied Basic Research Project of Yunnan Province (Grant No. 2019FD051), Scientific Research Foundation of Education Department of Yunnan Province (Grant No. 2019J0047), and the Beijing Key Laboratory of the Innovative Development of Functional Staple and the Nutritional Intervention for Chronic Disease.

\section{References}

[1] T. Church and C. K. Martin, "The obesity epidemic: a consequence of reduced energy expenditure and the uncoupling of energy intake?” Obesity, vol. 26, no. 1, pp. 14-16, 2018.

[2] N. N. El-Agroudy, A. Kurzbach, R. N. Rodionov et al., "Are lifestyle therapies effective for NAFLD treatment?" Trends in Endocrinology \& Metabolism, vol. 30, no. 10, pp. 701-709, 2019.

[3] S. A. Adefegha, "Functional foods and nutraceuticals as dietary intervention in chronic diseases; novel perspectives for health promotion and disease prevention," Journal of Dietary Supplements, vol. 15, no. 6, pp. 977-1009, 2018.

[4] D. Del Rio, A. Rodriguez-Mateos, J. P. E. Spencer, M. Tognolini, G. Borges, and A. Crozier, "Dietary (Poly) phenolics in human health: structures, bioavailability, and evidence of protective effects against chronic diseases," 
Antioxidants \& Redox Signaling, vol. 18, no. 14, pp. 1818-1892, 2013.

[5] Y.-J. Kwon, H.-S. Lee, J.-W. Lee et al., "Association of carbohydrate and fat intake with metabolic syndrome," Clinical Nutrition, vol. 37, no. 2, pp. 746-751, 2018.

[6] Q. F. Zhou, J. X. Zhou, X. J. Liu et al., "Digestive enzyme inhibition of different phenolic fractions and main phenolic compounds of ultra-high-pressure-treated palm fruits: interaction and molecular docking analyses," Journal of Food Quality, vol. 2020, Article ID 8811597, 2020.

[7] Y. Zheng, J. Tian, W. Yang et al., "Inhibition mechanism of ferulic acid against $\alpha$-amylase and $\alpha$-glucosidase," Food Chemistry, vol. 317, Article ID 126346, 2020.

[8] U. Hossain, A. K. Das, S. Ghosh, and P. C. Sil, "An overview on the role of bioactive $\alpha$-glucosidase inhibitors in ameliorating diabetic complications," Food and Chemical Toxicology, vol. 145, no. 2, Article ID 111738, 2020.

[9] C. Proença, D. Ribeiro, M. Freitas, and E. Fernandes, "Flavonoids as potential agents in the management of type 2 diabetes through the modulation of $\alpha$-amylase and $\alpha$-glucosidase activity: a review," Critical Reviews in Food Science and Nutrition, pp. 1-71, 2021.

[10] S. Cai, O. Wang, M. Wang et al., "In vitro inhibitory effect on pancreatic lipase activity of subfractions from ethanol extracts of fermented oats (Avena sativa L.) and synergistic effect of three phenolic acids," Journal of Agricultural and Food Chemistry, vol. 60, no. 29, pp. 7245-7251, 2012.

[11] S. Li, J. Pan, X. Hu, Y. Zhang, D. Gong, and G. Zhang, "Kaempferol inhibits the activity of pancreatic lipase and its synergistic effect with orlistat," Journal of Functional Foods, vol. 72, Article ID 104041, 2020.

[12] R. Huang, Y. Zhang, S. Shen et al., "Antioxidant and pancreatic lipase inhibitory effects of flavonoids from different citrus peel extracts: an in vitro study," Food Chemistry, vol. 326, Article ID 126785, 2020.

[13] M. J. Rahman, P. Ambigaipalan, and F. Shahidi, "Biological activities of camelina and sophia seeds phenolics: inhibition of LDL oxidation, DNA damage, and pancreatic lipase and $\alpha$-glucosidase activities," Journal of Food Science, vol. 83, no. 3, pp. 237-245, 2018.

[14] J. Gao, B. Liu, Z. Ning, R. Zhao, A. Zhang, and Q. Wu, "Characterization and antioxidant activity of flavonoid-rich extracts from leaves of Ampelopsis grossedentata," Journal of Food Biochemistry, vol. 33, no. 6, pp. 808-820, 2009.

[15] Y. Chen, E. Wang, Z. Wei, Y. Zheng, R. Yan, and X. Ma, "Phytochemical analysis, cellular antioxidant and $\alpha$-glucosidase inhibitory activities of various herb plant organs," Industrial Crops and Products, vol. 141, Article ID 111771, 2019.

[16] J. Chen, Y. Wu, J. Zou, and K. Gao, “ $\alpha$-Glucosidase inhibition and antihyperglycemic activity of flavonoids from Ampelopsis grossedentata and the flavonoid derivatives," Bioorganic \& Medicinal Chemistry, vol. 24, no. 7, pp. 1488-1494, 2016.

[17] V. Spínola, E. J. Llorent-Martínez, and P. C. Castilho, "Inhibition of $\alpha$-amylase, $\alpha$-glucosidase and pancreatic lipase by phenolic compounds of Rumex maderensis (Madeira sorrel). Influence of simulated gastrointestinal digestion on hyperglycaemia-related damage linked with aldose reductase activity and protein glycation," $L W T$, vol. 118, Article ID 108727, 2020.

[18] Y. Jia, Y. Ma, G. Cheng, Y. Zhang, and S. Cai, "Comparative study of dietary flavonoids with different structures as $\alpha$-glucosidase inhibitors and insulin sensitizers," Journal of Agricultural and Food Chemistry, vol. 67, no. 37, pp. 1052110533, 2019.
[19] V. Kumar, S. Kumar, and P. Rani, "Pharmacophore modeling and 3D-QSAR studies on flavonoids as $\alpha$-glucosidase inhibitors," Der Pharma Chemica, vol. 2, no. 3, pp. 324-335, 2010.

[20] J. Yang, X. Wang, C. Zhang et al., "Comparative study of inhibition mechanisms of structurally different flavonoid compounds on $\alpha$-glucosidase and synergistic effect with acarbose," Food Chemistry, vol. 347, Article ID 129056, 2021.

[21] Y. F. Li, Y. Q. Chang, J. Deng et al., "Prediction and evaluation of the lipase inhibitory activities of tea polyphenols with 3DQSAR models," Scientific Reports, vol. 6, no. 1, pp. 34387-34414, 2016.

[22] Y. S. Hamed, M. Abdin, A. M. Rayan, H. M. Saleem Akhtar, and X. Zeng, "Synergistic inhibition of isolated flavonoids from Moringa oleifera leaf on $\alpha$-glucosidase activity," LWT, vol. 141, Article ID 111081, 2021.

[23] L. Han, C. Fang, R. Zhu, Q. Peng, D. Li, and M. Wang, "Inhibitory effect of phloretin on $\alpha$-glucosidase: kinetics, interaction mechanism and molecular docking," International Journal of Biological Macromolecules, vol. 95, pp. 520-527, 2017.

[24] X. Wu, H. Ding, X. Hu et al., "Exploring inhibitory mechanism of gallocatechin gallate on a-amylase and a-glucosidase relevant to postprandial hyperglycemia," Journal of Functional Foods, vol. 48, pp. 200-209, 2018.

[25] M. Taha, F. Rahim, K. Zaman et al., "Synthesis, $\alpha$-glycosidase inhibitory potential and molecular docking study of benzimidazole derivatives," Bioorganic Chemistry, vol. 95, Article ID 103555, 2020.

[26] H. Tang, F. Ma, D. Zhao, and Z. Xue, "Exploring the effect of salvianolic acid $\mathrm{C}$ on $\alpha$-glucosidase: inhibition kinetics, interaction mechanism and molecular modelling methods," Process Biochemistry, vol. 78, pp. 178-188, 2019. 